\title{
Fluoxetine for motor recovery after acute intracerebral hemorrhage (FMRICH): study protocol for a randomized, double-blind, placebo-controlled, multicenter trial
}

Juan Manuel Marquez-Romero ${ }^{1,2^{*}}$, Antonio Arauz ${ }^{2}$, José Luis Ruiz-Sandoval ${ }^{3}$, Erick de la Cruz-Estrada ${ }^{4}$, Maria Raquel Huerta-Franco ${ }^{5}$, Gerónimo Aguayo-Leytte ${ }^{6}$, Angélica Ruiz-Franco ${ }^{2}$ and Humberto Silos ${ }^{2}$

\begin{abstract}
Background: Spontaneous, nontraumatic intracerebral hemorrhage $(\mathrm{ICH})$ is a subtype of stroke that causes a great amount of disability and economic and social burden. This is particularly true in developing countries where it accounts for between $20 \%$ and $50 \%$ of all strokes. Pharmacological and surgical interventions have been attempted to reduce the mortality and disability caused by $\mathrm{ICH}$, with unsuccessful results. Recently, the use of fluoxetine in addition to physical rehabilitation has been proven useful to improve motor recovery following cerebral infarct. The purpose of this study is to test whether a 3-month treatment with fluoxetine enhances motor recovery in nondepressed patients with acute intracerebral hemorrhage.

Methods/design: Our study is a randomized, double-blind, placebo-controlled, multicenter clinical trial. We will recruit 86 patients with intracerebral hemorrhage of both sexes, aged $>18$ years, from four Mexican hospitals. The patients will receive either $20 \mathrm{mg}$ of fluoxetine or a placebo once daily for 90 days. The primary outcome is the mean change in the Fugl-Meyer Motor Scale score between inclusion (day 0) and day 90. The secondary outcomes will be changes in the Barthel Index, the Modified Rankin scale and the National Institutes of Health stroke scale. The outcomes will be measured at day $42 \pm 7$ days and at day 90 , for a total of four visits with each subject (at screening and at 0,42 and 90 days).

Discussion: Current guidelines recommend early supported hospital discharge and home-based rehabilitation programs as the only cost-effective intervention to aid the recovery of patients with intracerebral hemorrhage. Nevertheless, such interventions are dependent on available resources and funding, which make them very difficult to implement in developing countries. We believe that the identification of a helpful pharmacological intervention to aid the motor recovery of these patients will constitute a breakthrough that will have a major impact in reducing the burden of disease caused by this subtype of stroke worldwide, especially in the developing world.
\end{abstract}

Trial registration: Current Controlled Trials NCT01737541

Keywords: Intracerebral hemorrhage, Stroke, Motor recovery, Fluoxetine, Randomized controlled trial

\footnotetext{
*Correspondence: scint1st@gmail.com

${ }^{1}$ Centro de Ciencias de la Salud, Universidad Autónoma de Aguascalientes,

Av. Universidad \# 940, Ciudad Universitaria, Aguascalientes, 20131, Mexico

${ }^{2}$ Stroke Clinic, Instituto Nacional de Neurología y Neurocirugía "MVS", Av.

Insurgentes Sur \# 3877, Col. La Fama, Mexico City, 14269, Mexico

Full list of author information is available at the end of the article
} 


\section{Background}

Spontaneous, nontraumatic intracerebral hemorrhage (ICH) is a significant cause of morbidity and mortality worldwide, and no treatment has been proven effective [1]. Each year, approximately 1 million people suffer from ICH [2], which causes a great amount of disability and economic and social burden. This is particularly true in developing countries where ICH accounts for between $20 \%$ and $50 \%$ of all strokes [3]. Hispanic Americans and especially Mexican Americans have been shown to have an increased risk of ICH compared with non-Hispanic whites [4]. The higher prevalence of $\mathrm{ICH}$ appears to be very similar in Hispanic patients living in Mexico and in the USA [5]. Despite these data, it is not clear whether this excess in incidence is due to differences inherent to ethnicity, or due to other differences in the disease pathogenesis.

Several therapeutic approaches have been attempted to reduce the burden in terms of death and disability from $\mathrm{ICH}$, including pharmacological and surgical interventions, but trials have been largely unsuccessful [6]. These failures have encouraged a focus on management in intensive care units and physical rehabilitation as the mainstream approaches to reduce mortality and disability, respectively [7].

Recently, the use of antidepressant drugs as an adjunctive treatment to augment recovery in stroke patients has been studied in small clinical trials [8-11]. In 2011, Chollet and colleagues [12] tested adjunctive treatment with fluoxetine in addition to physical rehabilitation to improve motor recovery from cerebral infarct (CI), with excellent results. Subsequent evidence indicated that this improvement is persistent through time [13].

It is thus necessary to elucidate the relative importance and potential benefits of the use of fluoxetine in ICH patients, so that this drug can play a role as an adjunctive therapy to aid the motor recovery of ICH patients, similar to CI patients.

This study will be the first reported randomized, double-blind, placebo-controlled clinical trial of the use of fluoxetine for motor recovery in patients with $\mathrm{ICH}$. The main purpose of this study is to test whether a three-month treatment with fluoxetine enhances motor recovery in nondepressed patients with acute intracerebral hemorrhage.

\section{Methods/design}

\section{Objectives and hypothesis Objectives}

The main objectives of this trial are to (1) compare the magnitude of motor recovery, measured with the FuglMeyer Motor Scale (FMMS), in patients receiving fluoxetine with that of patients receiving placebo, and (2) establish the relationship between motor recovery and functional recovery as measured with the Barthel index
(BI), the modified Rankin scale and the National Institutes of Health stroke scale (NIHSS).

\section{Hypothesis}

We hypothesize that the mean change in FMMS between inclusion (day 0) and day 90 will be significantly higher in the patients receiving fluoxetine compared with those receiving placebo.

\section{Study design and period}

This study is a 12-week randomized, double-blind, placebocontrolled, multicenter trial at four Mexican hospitals. Figure 1 shows the schematic flow of the study.

\section{Study groups}

The study will include the following two arms: the fluoxetine group (treatment arm) and the placebo group (control arm).

\section{Population}

The subjects will be patients of both sexes with acute $\mathrm{ICH}$ and ages above 18 years.

\section{Eligibility criteria}

Inclusion Criteria

1. Men and women ages $\geq 18$ years

2. Individuals who meet one of the following criteria: 2.1. Patients who had an acute intracerebral hemorrhage within the past 10 days causing hemiparesis or hemiplegia

2.2. Fugl-Meyer motor scale (FMMS) scores $\leq 55$

3. Written, informed consent for participation in the trial

\section{Exclusion Criteria}

1. Severe post-stroke disability (National Institutes of Health stroke scale [NIHSS] score $>20$ )

2. Premorbid disability, evidenced by residual motor deficit from a previous stroke

3. Comprehension deficits or severe aphasia

4. Previous diagnosis of depression or one of the following:

4.1. Hospital anxiety and depression scale (HAD) score $\geq 11$ points

4.2. Taking antidepressant drugs two weeks before inclusion

5. Taking neuroleptic drugs or benzodiazepines 2 weeks before inclusion

6. Other major diseases with life expectancy $\leq 3$ months.

Withdrawal Criteria

1. Detection of eligibility violations 


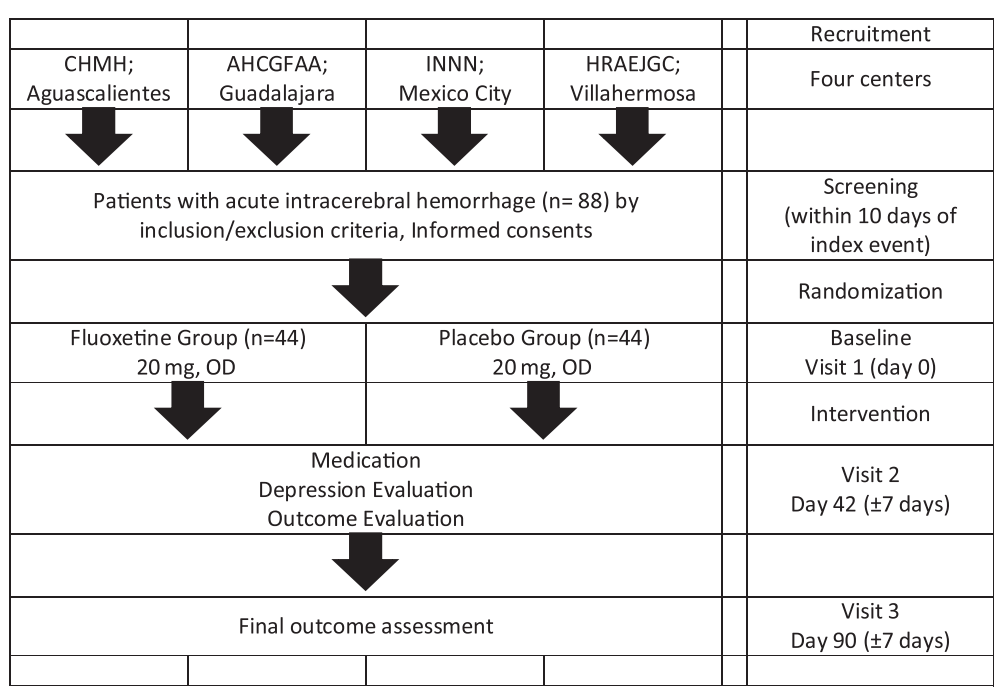

Figure 1 Flowchart of the fluoxetine for motor recovery after acute intracerebral hemorrhage (FMRICH) clinical trial.

2. Poor compliance $(<90 \%)$ or noncompliance

3. Use of any medication or treatment during the trial that could affect the study results

4. Occurrence of a serious adverse event

4.1Subject has an acute reaction (allergy, shock) to the investigational product

4.2Subject develops depression, evidenced by HAD score $\geq 11$ points at Visit 2

5. Subject withdraws consent or is uncooperative

The patients withdrawn after randomization will be followed for outcomes

\section{Interventions}

Participating subjects assigned to either the treatment group or the placebo group will be instructed to take a pill of fluoxetine $(20 \mathrm{mg}$ ) or placebo once daily for 90 days. Fluoxetine and placebo tablets will be identical in form, color, odor and packaging.

\section{Concomitant treatments and forbidden drugs}

The product name, use, dosage and duration of all medications used by the patients during their hospital stay and follow-up will be recorded. Only the use of antidepressants will be prohibited.

\section{Sample size calculation}

A sample size of 35 in each group will be sufficient to detect a clinically important difference of 14.5 points on the FMMS, assuming a standard deviation of 21.3 FMMS points on the basis of the findings of the fluoxetine in motor recovery of patients with acute ischaemic stroke (FLAME) trial [12], using a two tailed $t$-test of the difference between means, a power of $80 \%$, and a significance level of $5 \%$. This number has been increased to 44 per group (total of 88 ), to allow for a predicted $20 \%$ loss to follow-up.

The formula for the sample size for comparison of two means (two-sided) is as follows:

$$
\begin{aligned}
& n=\frac{2 \sigma^{2}\left(Z_{a}+Z_{b}\right)^{2}}{\delta^{2}} \\
& Z_{a}=1.96 \\
& Z_{b}=0.84
\end{aligned}
$$

$\delta=$ size of difference of clinical importance $=14.5$

$\sigma=$ standard deviation of the primary outcome variable $=21.3$

$$
n=\frac{2 * 21.3^{2}(1.96+0.84)^{2}}{14.5^{2}} \approx 35
$$

Adjusted for $20 \%$ patients lost to follow-up:

$$
n_{c}=\frac{n}{1-l} \approx 44
$$

$l=$ expected frequency of patients lost to follow-up $=0.20$

\section{Randomization method}

A pharmaceutical laboratory (Psicofarma ${ }^{\text {TM }}$ S.A. de C.V.) will be responsible for the manufacture and randomization of the investigational product, which will be achieved using a web-based randomization program. This program will be set to assign participants equally to each site at a ratio of $1: 1$.

Each of the sites will be assigned 22 participants. The manufacturer will then deliver the pre-randomized bottles 
containing the investigational product to each recruiting center.

Study subjects who satisfy the eligibility criteria at each recruiting center will receive the investigational product corresponding to a consecutive number assigned according to their entrance to the study.

\section{Blinding}

Both the investigator and the subject will be blinded to the assignment of the study drugs. The manufacturer of the tablets will label the investigational drugs by the randomization code number. The labeled experimental products will be provided to the recruiting centers by the manufacturer. An envelope containing all randomization codes will be delivered to the principal investigator and will be kept sealed until the conclusion of the trial.

\section{Recruitment}

Participants will be recruited at the emergency departments at four Mexican hospitals:

The Centenario Hospital 'Miguel Hidalgo' (located in Aguascalientes, a midsized city in central México), the Instituto Nacional de Neurología y Neurocirugía (located in Mexico City), the Antiguo Hospital Civil de Guadalajara 'Fray Antonio Alcalde' (located in Guadalajara, a major city in western Mexico) and the Hospital Regional de Alta Especialidad 'Dr. Juan Graham Casassus' (located in Villahermosa, a midsized city in the south of Mexico).

\section{Study schedule}

The measurements that will be carried out at each visit are listed in Table 1.

\section{Measurement tools Questionnaires}

\section{The fugl-meyer assessment (FMA)}

The Fugl-Meyer assessment (FMA) [14] is a stroke-specific, performance-based impairment index. It is designed to assess motor functioning, balance, sensation and joint functioning in hemiplegic post-stroke patients. Each of the five FMA domains can be separated to test a specific construct. The motor domain (FMMS) includes items assessing movement, coordination and reflex action of the shoulder, elbow, forearm, wrist, hand, hip, knee, and ankle. We will use the FMMS to assess the motor recuperation of patients in this study.

\section{The Barthel index}

The Barthel index [15] is a widely used measure of functional disability. The index was developed for use in the rehabilitation of patients with stroke. This index measures the extent to which someone can function independently and has the mobility necessary for their daily life activities.
Table 1 Study schedule of fluoxetine for motor recovery after acute intracerebral hemorrhage (FMRICH) clinical trial

\begin{tabular}{|c|c|c|c|c|}
\hline & Screening & $\begin{array}{c}\text { Visit } \\
1\end{array}$ & $\begin{array}{c}\text { Visit } \\
2\end{array}$ & $\begin{array}{c}\text { Visit } \\
3\end{array}$ \\
\hline Informed consent & - & & & \\
\hline Inclusion criteria & • & & & \\
\hline Demographics $^{a}$ & $\cdot$ & & & \\
\hline Inclusion/exclusion criteria check & $\cdot$ & & & \\
\hline Vital signs ${ }^{b}$ & & $\cdot$ & $\cdot$ & $\cdot$ \\
\hline Medical/drug use history ${ }^{c}$ & & • & & \\
\hline Smoking/drinking status & & • & & \\
\hline Laboratory tests $^{\mathrm{d}}$ & & $\cdot$ & & \\
\hline Lipid test ${ }^{\mathrm{e}}$ & & - & & \\
\hline Coagulation tests $^{f}$ & & • & & $\cdot$ \\
\hline Date of bleeding & & $\cdot$ & & \\
\hline $\begin{array}{l}\text { Condition associated with } \\
\text { bleeding }^{9}\end{array}$ & & $\cdot$ & & \\
\hline $\mathrm{BCT}$ & $\cdot$ & & & \\
\hline Date of BCT & $\cdot$ & & & \\
\hline Volume of the hemorrhage ${ }^{h}$ & - & & & \\
\hline Localization of the hemorrhage & $\cdot$ & & & \\
\hline Concomitant medication & $\cdot$ & - & $\cdot$ & $\cdot$ \\
\hline Adverse event & & & $\cdot$ & $\cdot$ \\
\hline NHISS & & - & $\cdot$ & - \\
\hline Depression Scale & $\cdot$ & & $\cdot$ & $\cdot$ \\
\hline Fugl-Meyer Motor Scale & - & & $\cdot$ & - \\
\hline Patient's rehabilitation log & & & - & - \\
\hline Barthel Index & & $\cdot$ & $\cdot$ & $\cdot$ \\
\hline Modified Rankin Scale & & $\cdot$ & • & $\cdot$ \\
\hline Pill count & & & • & • \\
\hline
\end{tabular}

${ }^{\mathrm{a} A g e}$, gender, educational level, marital status, occupational status, date of birth, contact address and telephone number. ${ }^{\text {b } B l o o d ~ p r e s s u r e ~}(\mathrm{mmHg})$, pulse (beats/minute) and body temperature $\left({ }^{\circ} \mathrm{C}\right)$. ' $D$ iabetes Mellitus, hypertension, dyslipidemia. ${ }^{\mathrm{d}}$ Hemoglobin, hematocrit, red blood cell count, white blood cell count, platelet count, blood urea, nitrogen/creatinine ratio, fasting plasma glucose. ${ }^{\mathrm{e}}$ Total cholesterol (mg/dl), high-density lipoprotein cholesterol $(\mathrm{mg} / \mathrm{dl})$, low-density lipoprotein cholesterol $(\mathrm{mg} / \mathrm{dl})$, very low-density lipoprotein cholesterol (mg/dl), triglyceride $(\mathrm{mg} / \mathrm{dl})$. International Normalized Ratio, prothrombin time, activated partial thromboplastic time. ${ }^{9}$ Trauma, Drugs, bleeding disorder, exercise. ${ }^{\mathrm{h}} \mathrm{ABC} / 2$ method. $\mathrm{BCT}$, Brain Computed Tomography; NIHSS, National Institutes of Health Stroke Scale.

We will use the BI to establish the relationship between motor recovery (FMMS) and functional recovery.

\section{The hospital anxiety and depression scale}

The hospital anxiety and depression scale (HAD) [16] is a scale widely used to evaluate anxiety and depression in hospitalized patients and has been validated in Spanish [17]. It consists of 14 items: the odd items represent the anxiety subscale and the even items the depression subscale, and both are scored between 0 and 3. The total score is 
obtained by the summation of all items in each subscale. The severity of the condition increases with the score. In a validation study by Abent et al. [18], the use of the HAD scale resulted in a sensitivity of no less than 91.7 (specificity: 65.3) using the optimum threshold score of 11 . The authors concluded that although there were no substantial differences in the performances of the four depression rating scales that they studied, the HAD scale was preferred in screening for post-stroke depression because it requires the least amount of time to administer. Thus, we will use the HAD to rule out depression in the patients enrolling and participating in this study.

\section{Compliance}

All subjects will be asked to return the remaining tablets at their next visit. The rate of compliance (percentage) will be calculated on the basis of the returned tablets.

Compliance $(\%)=100$ - returned tablets/expected intake $\times 100$.

Investigational drugs will be distributed in packs of 50 at each visit.

$50=1$ times $/$ day $\times 7$ days $/$ week $\times 6$ weeks $/$ visit +8 extras.

\section{Outcomes}

Both primary and secondary end points will be measured at each visit according to the study schedule (Table 1).

\section{Primary outcome}

The primary outcome is the mean change in FMMS score between inclusion (day 0) and day 90.

\section{Secondary outcomes}

Both within-group and between-group analyses will be performed for each outcome. The differences in the following variables between the baseline (visit 1) and the last visit (visit 3) will be calculated.

1. Barthel Index

2. Modified Rankin scale

3. NIHSS

\section{Statistical analysis \\ Efficacy assessment}

The primary and secondary outcomes will be analyzed using the intention to treat (ITT) method. The full analysis set for the ITT method will include all randomized subjects, regardless of their subsequent withdrawal after enrollment. Continuous variables will be reported as the means $\pm \mathrm{SD}$, and categorical variables will be reported as percentages. The Kolmogorov-Smirnoff test will be used to test normality for the continuous variables, and baseline characteristics will be compared by either Student's $t$-test/U Mann-Whitney for continuous variables or the $\chi^{2}$ test (Fisher's exact test when the expected value is $<5$ ) for categorical data.

The primary analysis for efficacy (full-analysis set) will consist of a comparison of the change in FMMS scores at 90 days. To handle the missing data derived from withdrawal and lost to follow-up patients, the last available outcome measure value obtained from the withdrawn/lost to follow-up patient will be used for the analysis (last observation carried forward (LOCF) strategy). A multiple linear regression analysis will be performed to control for baseline factors that show an imbalance.

For the within-group analyses, primary and secondary outcome variables will be evaluated by a paired t-test. Alternatively, for non-normally distributed data, a Wilcoxon test will be performed. Analysis of covariance will be used to analyze differences in each group, adjusting for age and NIHSS as covariates. Statistical significance will be defined as $\mathrm{P}<0.05$. SPSS for Windows version 17.0 software (SPSS, Inc, Chicago, IL, USA) will be used for the analyses.

\section{Adverse event reporting}

Adverse events (AEs) will be recorded in medical diagnostic terminology. Detailed symptoms, duration, severity, causal relationships, actions taken, results and other information will be recorded for each AE. All AEs must be observed and recorded in the case report file $(\mathrm{CRF})$ in the $\mathrm{AE}$ report section.

\section{Data quality control, data collection and data management}

Data quality control will be achieved through monitoring during the trial. After checking the written CRF, welltrained clinical research associates of the principal investigator will collect the data.

\section{Ethical issues}

This study has been approved by the institutional review boards (IRBs) at the Centenario Hospital 'Miguel Hidalgo', the Antiguo Hospital Civil de Guadalajara 'Fray Antonio Alcalde' and the Hospital Regional de Alta Especialidad 'Dr. Juan Graham Casassus', and by the Bioethics Committee (reference: Oficio No. $\mathrm{CB} / 304 / 11$ ) and the Scientific Committee (reference: Oficio No. DIC/547/11) of the Instituto Nacional de Neurología y Neurocirugía.

Written informed consent will be obtained from each individual prior to enrollment. Research will be performed in compliance with the Helsinki Declaration and with the Good Clinical Practice Guidelines.

\section{Discussion}

The degree of recovery and the speed of recovery from disabilities secondary to $\mathrm{ICH}$ are different from the recovery of patients with $\mathrm{CI}$, although this difference has rarely been documented $[19,20]$. This is partly because the evidence 
comes from countries where there are a disproportionately lower number of patients with $\mathrm{ICH}$ compared with $\mathrm{CI}$ available to extract adequate conclusions. However, the existing evidence appears to demonstrate that patients with $\mathrm{ICH}$ recover faster and to a quantitatively greater degree compared with patients with CI. For example, in the study by Katrak et al. [21], even though the studied patients with ICH had a greater level of disability at discharge, they achieved significantly greater gains in function than patients with CI after rehabilitation. This was the case regardless of the severity of disability on admission.

The study by Nannetti et al. [22], in which $20 \%$ of the studied stroke patients suffered from $\mathrm{ICH}$, patients receiving antidepressants (mostly SSRIs) made the most rapid recoveries during the hospital stay period. However, lower levels of improvement were seen after discharge, which the authors attribute to the fact that the antidepressant treatment was not continued after discharge by some patients (20.4\%). This recovery was observed in the group of patients with post stroke depression (PSD) as well as in those without PSD.

The aforementioned data indicate there is a greater amount of potential motor recovery for patients with ICH compared with those with CI. Still, approximately half of ICH survivors will remain dependent on others for daily life activities [23]. The motor recovery mechanisms after stroke are still being elucidated, and most of the studies have focused on patients with CI, so little is known about the motor recovery mechanism for patients with ICH [24]. It has been demonstrated that the presence of wallerian degeneration in the pyramidal tract remote from the initial lesion on magnetic resonance (MR) images correlates with a poor outcome both in $\mathrm{CI}$ [25] and in $\mathrm{ICH}$ [26] and that the primary motor cortex (M1) plays a key role in the motor recovery of stroke patients. Strategies aimed to facilitate activity in the ipsilesional M1 or to downregulate activity in the contralesional M1 have been found to be the most useful [27]. This ipsilesional facilitation/contralesional downregulation has been attempted by modulation of somatosensory input originating in the paretic or healthy hands. Ipsilesional facilitation/contralesional downregulation is also one of the apparent mechanisms of action of selective serotonin re-uptake inhibitors (SSRIs) in the motor recovery of stroke patients, in addition to cortical reorganization and changes in the somatotopy of M1 [28].

Current guidelines recommend early supported hospital discharge and home-based rehabilitation programs as the only cost-effective intervention to aid in the recovery of $\mathrm{ICH}$ patients [1]. Such interventions are highly dependent on available resources and funding, which make them very difficult to implement in developing countries.
In Mexico, $\mathrm{ICH}$ accounts for nearly 30\% of all stroke cases and produces significant functional disability and death, with $30 \%$ mortality and another $30 \%$ of patients severely disabled (modified Rankin scale $>3$ ) [29]. Up to $25 \%$ of Mexican family caregivers have to stop or cut back on work to care for stroke survivors [30]. We strongly believe that identification of a pharmacological intervention to aid in the motor recovery of patients with $\mathrm{ICH}$ will constitute a breakthrough that will have a major impact on the burden of disease derived from $\mathrm{ICH}$ in our country. We also theorize that the effect size should be bigger than that found in patients with $\mathrm{CI}$.

\section{Trial status}

The trial was first designed in 2011, and subject recruitment began in November 2012.

\section{Abbreviations}

AE: adverse event; AHCGFAA: Antiguo Hospital Civil de Guadalajara 'Fray Antonio Alcalde'; BCT: brain computed tomography; BI: Barthel index; CHMH: Centenario Hospital 'Miguel Hidalgo'; Cl: cerebral infarct; CRF: case report file; FLAME: fluoxetine in motor recovery of patients with acute ischaemic stroke; FMA: Fugl-Meyer assessment; FMMS: Fugl-Meyer motor scale; HAD: hospital anxiety and depression scale; HRAEJGC: Hospital Regional de Alta Especialidad 'Dr. Juan Graham Casassus'; ICH: intracerebral hemorrhage; ITT: intention-to-treat; INNN: Instituto Nacional de Neurología y Neurocirugía; LOCF: last observation carried forward; M1: primary motor cortex; MR: magnetic resonance; NIHSS: National Institutes of Health stroke scale; PSD: post-stroke depression; SSRIs: selective serotonin re-uptake inhibitors.

\section{Competing interests}

The authors declare that they have no competing interests.

\section{Authors' contributions}

JMM is the Chief Investigator for FMRICH and leads the project. AA is the trial director and was responsible for the initial development of the protocol along with JMM. MRH advised on the statistical, sample size and data analysis sections. JMM coordinated the development of trial documentation and ethics approval, and prepared and edited this article for journal publication. JLR, GA, EC, AR, HS are all co-investigators on the project and have made contributions to developing the protocol prior to the start of the trial. All authors have commented on this article. All authors read and approved the final manuscript.

\section{Acknowledgements}

This project is possible due to the unconstrained support from Psicofarma ${ }^{\mathrm{TM}}$ S.A. de C.V. The company played no role in the conception, development, and implementation of the trial.

\section{Author details}

${ }^{1}$ Centro de Ciencias de la Salud, Universidad Autónoma de Aguascalientes, Av. Universidad \# 940, Ciudad Universitaria, Aguascalientes, 20131, Mexico. 2Stroke Clinic, Instituto Nacional de Neurología y Neurocirugía "MVS", Av. Insurgentes Sur \# 3877, Col. La Fama, Mexico City, 14269, Mexico. ${ }^{3}$ Hospital Civil de Guadalajara "Fray Antonio Alcalde". Hospital 278, Col. El Retiro, Torre de Especialidades, 8vo. piso, Universidad de Guadalajara Guadalajara, Jalisco, 44280, Mexico. "Hospital De Alta Especialidad "Dr. Juan Graham Casasus". Carretera a la Isla SN. Col. Miguel Hidalgo, Villahermosa, Tabasco, Mexico. ${ }^{5}$ Department of Applied Sciences to Work, Health Science Division, University of Guanajuato, 37320 Leon, Guanajuato, Mexico. ${ }^{6}$ Centenario Hospital "Miguel Hidalgo", Galeana Sur 465. Col. Obraje, Aguascalientes, Mexico. 


\section{References}

1. Morgenstern LB, Hemphill JC 3rd, Anderson C, Becker K, Broderick JP, Connolly ES Jr, Greenberg SM, Huang JN, MacDonald RL, Messe SR, Mitchell PH, Selim M, Tamargo RJ, American Heart Association Stroke Council and Council on Cardiovascular Nursing: Guidelines for the management of spontaneous intracerebral hemorrhage: a guideline for healthcare professionals from the American Heart Association/American Stroke Association. Stroke 2010, 41:2108-2129.

2. Qureshi Al, Tuhrim S, Broderick JP, Batjer HH, Hondo H, Hanley DF: Spontaneous intracerebral hemorrhage. N Engl J Med 2001, 344:1450-1460.

3. Saposnik G, Del Brutto OH: Stroke in South America: a systematic review of incidence, prevalence, and stroke subtypes. Stroke 2003, 34:2103-2107.

4. Zahuranec DB, Brown DL, Lisabeth LD, Gonzales NR, Longwell PJ, Eden SV, Smith MA, Garcia NM, Morgenstern LB: Differences in intracerebral hemorrhage between Mexican Americans and non-Hispanic whites. Neurology 2006, 66:30-34.

5. Romano JG, Arauz A, Koch S, Dong C, Marquez JM, Artigas C, Merlos M, Hernandez B, Roa LF, Rundek T, Sacco RL: Disparities in Stroke Type and Vascular Risk Factors Between 2 Hispanic Populations in Miami and Mexico City. J Stroke Cerebrovasc Dis 2012. Epub ahead of print.

6. Steiner T, Petersson J, Al-Shahi Salman R, Christensen H, Cordonnier C, Csiba L, Harnof S, Krieger D, Mendelow D, Molina C, Montaner J, Overgaard K, Roine RO, Schmutzhard E, Tatlisumak T, Toni D, Stapf C, European Research Network on Intracerebral Haemorrhage: European research priorities for intracerebral haemorrhage. Cerebrovasc Dis 2011, 32:409-419.

7. Staykov D, Huttner HB, Kohrmann M, Bardutzky J, Schellinger PD: Novel approaches to the treatment of intracerebral haemorrhage. Int I Stroke 2010, 5:457-465.

8. Dam M, Tonin P, De Boni A, Pizzolato G, Casson S, Ermani M, Freo U, Piron $L$, Battistin $L$ : Effects of fluoxetine and maprotiline on functional recovery in poststroke hemiplegic patients undergoing rehabilitation therapy. Stroke 1996, 27:1211-1214.

9. Pariente J, Loubinoux I, Carel C, Albucher JF, Leger A, Manelfe C, Rascol O, Chollet F: Fluoxetine modulates motor performance and cerebral activation of patients recovering from stroke. Ann Neurol 2001. 50:718-729.

10. Zittel S, Weiller C, Liepert J: Citalopram improves dexterity in chronic stroke patients. Neurorehabil Neural Repair 2008, 22:311-314.

11. Acler M, Robol E, Fiaschi A, Manganotti P: A double blind placebo RCT to investigate the effects of serotonergic modulation on brain excitability and motor recovery in stroke patients. J Neurol 2009, 256:1152-1158.

12. Chollet F, Tardy J, Albucher JF, Thalamas C, Berard E, Lamy C, Bejot Y, Deltour S, Jaillard A, Niclot P, Guillon B, Moulin T, Marque P, Pariente J, Arnaud C, Loubinoux I: Fluoxetine for motor recovery after acute ischaemic stroke (FLAME): a randomised placebo-controlled trial. Lancet Neurol 2011, 10:123-130.

13. Mikami K, Jorge RE, Adams HP Jr, Davis PH, Leira EC, Jang M, Robinson RG: Effect of antidepressants on the course of disability following stroke. Am $J$ Geriatr Psychiatr 2011, 19:1007-1015.

14. Fugl-Meyer AR, Jaasko L, Leyman I, Olsson S, Steglind S: The post-stroke hemiplegic patient. 1. a method for evaluation of physical performance. Scand J Rehabil Med 1975, 7:13-31.

15. Mahoney FI, Barthel DW: Functional Evaluation: The Barthel Index. Md State Med J 1965, 14:61-65.

16. Zigmond AS, Snaith RP: The hospital anxiety and depression scale. Acta Psychiatr Scand 1983, 67:361-370.

17. López-Roig S, Terol MC, Pastor-Mira MA, Neipp MC, Massutí B, RodríguezMarín J: Anxiety and Depression. The HAD scale validation in cancer patients. Revista de Psicología de la Salud 2000, 12:127-155.

18. Aben I, Verhey F, Lousberg R, Lodder J, Honig A: Validity of the beck depression inventory, hospital anxiety and depression scale, SCL-90, and hamilton depression rating scale as screening instruments for depression in stroke patients. Psychosomatics 2002, 43:386-393.

19. Kelly PJ, Furie KL, Shafqat S, Rallis N, Chang Y, Stein J: Functional recovery following rehabilitation after hemorrhagic and ischemic stroke. Arch Phys Med Rehabil 2003, 84:968-972.

20. Schepers VP, Ketelaar M, Visser-Meily AJ, de Groot V, Twisk JW, Lindeman E: Functional recovery differs between ischaemic and haemorrhagic stroke patients. J Rehabil Med 2008, 40:487-489.
21. Katrak PH, Black D, Peeva V: Do stroke patients with intracerebral hemorrhage have a better functional outcome than patients with cerebral infarction? PM R 2009, 1:427-433.

22. Nannetti L, Paci M, Pasquini J, Lombardi B, Taiti PG: Motor and functional recovery in patients with post-stroke depression. Disabil Rehabil 2005, 27:170-175.

23. Rost NS, Smith EE, Chang Y, Snider RW, Chanderraj R, Schwab K, FitzMaurice E, Wendell L, Goldstein JN, Greenberg SM, Rosand J: Prediction of functional outcome in patients with primary intracerebral hemorrhage: the FUNC score. Stroke 2008, 39:2304-2309.

24. Jang SH, Kim SH, Cho SH, Choi BY, Cho YW: Demonstration of motor recovery process in a patient with intracerebral hemorrhage. NeuroRehabilitation 2007, 22:141-145.

25. Ward NS, Newton JM, Swayne OB, Lee L, Thompson AJ, Greenwood RJ, Rothwell JC, Frackowiak RS: Motor system activation after subcortical stroke depends on corticospinal system integrity. Brain 2006, 129:809-819.

26. Cho SH, Kim SH, Choi BY, Kang JH, Lee CH, Byun WM, Jang SH: Motor outcome according to diffusion tensor tractography findings in the early stage of intracerebral hemorrhage. Neurosci Lett 2007, 421:142-146.

27. Sharma N, Cohen LG: Recovery of motor function after stroke. Dev Psychobiol 2012, 54:254-262.

28. Gonzenbach RR, Taegtmeyer AB, Luft A, Russmann S: Fluoxetine and motor recovery after ischaemic stroke. Lancet Neurol 2011, 10:499-500. author reply 500-501.

29. Ruiz-Sandoval IL Chiquete E, Garate-Carrillo A, Ochoa-Guzman A, Arauz A Leon-Jimenez C, Carrillo-Loza K, Murillo-Bonilla LM, Villarreal-Careaga J, Barinagarrementeria F, Cantu-Brito C: Spontaneous intracerebral hemorrhage in Mexico: results from a Multicenter Nationwide Hospital-based Registry on Cerebrovascular Disease (RENAMEVASC). Rev Neurol 2011, 53:705-712.

30. Ferri CP, Schoenborn C, Kalra L, Acosta D, Guerra M, Huang Y, Jacob KS, Llibre Rodriguez JJ, Salas A, Sosa AL, Williams JD, Liu Z, Moriyama T, Valhuerdi A, Prince MJ: Prevalence of stroke and related burden among older people living in Latin America, India and China. J Neurol Neurosurg Psychiatry 2011, 82:1074-1082.

doi:10.1186/1745-6215-14-77

Cite this article as: Marquez-Romero et al:: Fluoxetine for motor recovery after acute intracerebral hemorrhage (FMRICH): study protocol for a randomized, double-blind,

placebo-controlled, multicenter trial. Trials 2013 14:77.

\section{Submit your next manuscript to BioMed Central and take full advantage of:}

- Convenient online submission

- Thorough peer review

- No space constraints or color figure charges

- Immediate publication on acceptance

- Inclusion in PubMed, CAS, Scopus and Google Scholar

- Research which is freely available for redistribution 